\title{
Étudier la communication organisationnelle et santé des organisations de santé au health organizing
}

Studying the health and organizational communication of health organizations in health organizing

Maria Cherba et Consuelo Vásquez

\section{OpenEdition Journals}

Édition électronique

URL : http://journals.openedition.org/communicationorganisation/4598

DOI : 10.4000/communicationorganisation.4598

ISSN : $1775-3546$

Éditeur

Presses universitaires de Bordeaux

\section{Édition imprimée}

Date de publication : 1 juin 2014

Pagination : 223-242

ISBN : 978-2-86781-904-9

ISSN : $1168-5549$

\section{Référence électronique}

Maria Cherba et Consuelo Vásquez, «Étudier la communication organisationnelle et santé des organisations de santé au health organizing », Communication et organisation [En ligne], 45 | 2014, mis en ligne le 01 juin 2017, consulté le 30 avril 2019. URL : http://journals.openedition.org/ communicationorganisation/4598; DOI : 10.4000/communicationorganisation.4598 


\title{
Étudier la communication organisationnelle et santé des organisations de santé au health organizing
}

\author{
Maria Cherba' et Consuelo Vásquez²
}

\section{Introduction}

Les organisations de santé sont devenues dans les dernières décennies un objet d'étude privilégié pour plusieurs disciplines - telles la gestion, la psychologie, la sociologie - qui s'intéressent à la manière dont la santé s'organise dans des institutions et aux effets que ces formes organisationnelles peuvent avoir sur le travail, les acteurs, les normes, les technologies, etc.

La notion " organisations de santé " fait référence aux organisations de soins (comme les hôpitaux, les cliniques, les centres de soins) et aux organisations de promotion, de prévention et de gestion de la santé (ce que certains pourraient associer à la gouvernance de la santé), qu'elles soient privées ou publiques, internationales, nationales ou locales, à but lucratif ou non lucratif. Les organisations de santé ont été définies comme des bureaucraties professionnelles (Minztberg, 1995, 2001), des organisations pluralistes (Denis et al., 2001, 2007) ou des anarchies organisées (Denis et al., 1995), ou encore comme des organisations hautement fiables (Weick, 1995 ; Weick et Roberts, 1993 ; Weick et Sutcliffe, 2001). D’autres chercheurs les ont caractérisées par les logiques rationnelles qui articulent les processus décisionnels (Bercot, 2009 ; Ogien, 2009) ou par l'environnement surpeuplé de technologies (Bruni et al., 2010). Ces définitions mettent de l'avant la

\footnotetext{
1 Maria Cherba est étudiante au doctorat en communication à l'Université de Montréal. Elle s'intéresse aux enjeux communicationnels dans les milieux de la santé et est membre du Centre de recherche sur la communication et la santé (ComSanté) de l'Université du Québec à Montréal ; maria.cherba@umontreal.ca 2 Consuelo Vásquez ( $\mathrm{PhD}$, Université de Montréal) est professeure au Département de communication sociale et publique de l'Université du Québec à Montréal (UQÀM). Ses travaux s'inscrivent dans les approches constitutives de la communication organisationnelle, à partir desquelles elle aborde des questions associées aux organisations dites «fragiles » telles les organisations par projets et les organisations bénévoles. Elle est membre du groupe de recherche Langage, Organisation et Gouvernance de l'Université de Montréal. Elle fait aussi partie du Groupe d'Étude sur la Pratique de la Stratégie (GéPS) de HEC Montréal, de la Chaire en relations publiques et communication marketing et du Centre sur la communication et la santé (ComSanté) de l’UQÀM ; vasquez.consuelo@uqam.ca
} 
nature complexe, voire même évasive des organisations de santé, ce qui pose pour la recherche des défis théoriques et méthodologiques.

Cet article cherche à cerner la place et la contribution d'une approche centrée sur la communication organisationnelle pour étudier les organisations de santé. À l'instar de Grosjean et Lacoste (1999, p. 4) nous croyons qu'une meilleure compréhension de la dimension collective et communicationnelle peut apporter « des éclairages intéressants en matière de qualité et de fiabilité des soins, de conditions de travail, de management, de communication interne, d'implantation de nouvelles technologies ». Or, force est de constater que même si les initiatives se multiplient pour développer une telle approche, elles sont plutôt isolées. De plus, il n'existe pas de travaux qui dressent un portrait général de ce volet particulier de recherche en communication organisationnelle que nous nommons « communication organisationnelle et santé ».

Cet article se veut une première exploration des écrits sur la « communication organisationnelle et santé ». Notre objectif n'est pas de présenter un répertoire exhaustif, mais de cerner les deux questions suivantes: Quels sont les thèmes de recherche privilégiés par les chercheurs qui étudient les dynamiques organisationnelles et communicationnelles des organisations de santé ? Quel est l'apport théorique et empirique d'une approche de communication organisationnelle à l'étude des organisations de santé ?

Dans le texte qui suit, nous décrirons brièvement la méthodologie de recherche développée pour la recension des écrits, pour ensuite présenter les quatre thématiques qui ont émergé de l'analyse et les problématiques communicationnelles qui en découlent. En guise de conclusion, nous suggérerons quelques pistes de recherche permettant d'ouvrir la notion d'organisations de santé vers l'étude des processus organisant qui seraient constitutifs de ce type d'organisation, ou ce que Zoller (2010) nomme « health organizing ».

\section{Brève note méthodologique}

Le point de départ de cette recension furent deux colloques thématiques organisés dans le cadre de l'Association francophone pour le savoir : "Organisation et communication au sein des systèmes de santé " (ACFAS, 2009) et "Mutations des systèmes de santé et communication entre organisations, professionnels, patients et société civile »(ACFAS, 2010). Nous avons consulté les articles de ces actes de colloque et les références mentionnées dans les bibliographies.

Étant donné que notre étude s'ancre dans une réflexion sur l'apport d'une perspective communicationnelle, nous avons analysé ces recherches à partir d'une grille thématique développée par le Centre de recherche en communication et santé (ComSanté) de l'Université du Québec à Montréal qui repose sur les thèmes suivants : 1 ) coordination et collaboration aux niveaux 
intra- et inter-organisationnels ; 2) changement dans/des organisations de santé ; 3) gouvernance, régulation et modes de gestion ; 4) acteurs et identités professionnelles. En 2012, le centre ComSanté a organisé un colloque thématique «La santé à l'épreuve des reconfigurations organisationnelles et communicationnelles " (ACFAS, 2012), suivi par la parution d'un numéro spécial de la Revue internationale de la communication sociale et publique qui a aussi fait partie de notre recension (Vásquez et Jolivet, 2013).

Pour compléter cette première analyse de corpus, nous avons fait une recherche bibliographiques dans les bases de données en sciences sociales et sciences de la santé (PubMed, Sage et CAIRN.info), ainsi que dans quinze périodiques spécialisés dans le domaine. Afin de sélectionner les articles pertinents, nous nous sommes inspirées des qualités distinctives de la recherche en communication selon Carbaug et Buzzanel (2010), à savoir : 1) les phénomènes à l'étude sont conceptualisés en tant que phénomènes communicationnels ; 2) les questions de recherche portent sur les phénomènes de la communication (organisationnelle) ; 3) la méthodologie utilisée est basée sur une théorie en communication (organisationnelle) ; 4) les résultats de recherche sont interprétés à travers le prisme de la communication (organisationnelle), que ce soit sur le plan théorique ou pratique. La définition de la communication organisationnelle sur laquelle nous nous sommes basées est très ouverte et inclut à la fois la communication dans les organisations (la circulation de l'information) et la communication qui constitue les organisations (le langage et le discours) ; les interactions entre les personnes (les conversations, les réunions) et d'autres éléments comme les artefacts technologiques (Cooren et al., 2011).

Nous avons privilégié les recherches qualitatives, prometteuses pour étudier en profondeur les transformations complexes qu'ont connues les organisations de santé dans les dernières décennies (Caronna, 2010, p. 71-72). Au total, nous avons recensé plus de 90 travaux de recherche, articles, monographies, actes de colloques et communications scientifiques. Notons aussi qu'une grande partie des recherches recensées a été effectuée au Canada, en France et aux Etats-Unis, mais nous avons aussi analysé les travaux de chercheurs de l'Angleterre, de l'Italie, de l'Allemagne, des Pays-Bas.

Dans la section suivante, nous dresserons un portrait de recherches selon quatre axes thématiques (un tableau résumé se trouve en annexe). La distinction des axes répond à un effort analytique, et certains thèmes (comme la question des technologies de l'information et de la communication - TIC et les réseaux de santé) sont transversaux et peuvent être traités comme étant des vases communicants entre les quatre axes. 


\section{Thématiques de la communication organisationnelle et santé}

\section{Axe 1 : Coordination et collaboration aux niveaux intra- et inter-organisationnels}

Dans le contexte des changements institutionnels et technologiques auxquels font face les organisations de santé aujourd'hui, les besoins de coordination et de collaboration ne cessent de croître. Dans les travaux recensés, trois problématiques liées à ces enjeux ont été évoquées.

1. La constitution et la distribution des savoirs. Il ne s'agit pas uniquement des savoirs qui ont rapport à la profession médicale, mais aussi et surtout de savoir-faire communicationnel qui s'actualise dans les pratiques quotidiennes et les échanges informels (Grosjean, 2008 ; Lacoste, 2001). Les TIC en tant qu'outils de stockage et de transmission de données jouent un rôle important dans la constitution et le partage des savoirs et compétences collectifs, et contribuent ainsi au processus d'apprentissage organisationnel, tout en transformant les formes et les modes de collaboration (Ash et al., 2004 ; Benghozi, 2001 ; Bouzon, 2004 ; Grosjean et Bonneville, 2007 ; Pirnejad et al., 2008 ; Robey et Boudreau, 1999 ; Safran et al., 1998 ; Vitacca et al., 2009). Les auteurs s'entendent pour dire que les TIC encouragent à la fois la décentralisation (grâce aux outils permettant le travail à distance, comme le courrier électronique et le dossier patient électronique, DPE) et l'intégration (les systèmes informatisés exigent une rationalisation plus accrue et un contrôle plus rigoureux des processus de la création de l'information, de la transmission et de la rétroaction).

2. Le réseau comme mode spécifique de coordination des organisations de santé. La standardisation qui s'accompagne d'un plus grand niveau d'autonomie des acteurs (Grosjean et Bonneville, 2007) place les TIC au centre de l'évolution des réseaux de santé (Bourret, 2008). La notion de réseau fait référence à la fois à plusieurs organisations qui coopèrent ou à une organisation qui structure son activité en réseau (Benghozi et al., 2002) afin d'assurer la coordination de santé autour du patient, autour d'une pathologie, ou sur un espace géographique donné (Schweyer et al., 2004). Bourret (2010, p. 86 sqq.) conçoit le réseau de santé comme une " organisation d'interface " qui permet "la construction de la coopération et de la confiance entre tous les acteurs ». Nous observons aujourd'hui une multiplication de réseaux de santé : réseaux ville-hôpital en France (Bercot, 2006), Primary Care Trusts en Angleterre (Sheaff et al., 2003), réseaux locaux de services de santé et de services sociaux intégrés au Québec (Breton et al., 2008 ; Bonneville et Grosjean, 2009) ; et dans ce contexte la coordination et la recherche de cohérence inter-réseaux deviennent un enjeu majeur (Schweyer et al., 2004, p. 98) qui amène à questionner l'avenir des réseaux de santé (Bourret, 2008).

3. La négociation et les rapports de pouvoir. La coordination et la collaboration au sein des organisations de santé impliquent une négociation constante entre les acteurs multiples qui ont des expertises, des valeurs, des logiques 
organisationnelles différentes, voire contradictoires (Arber, 2008 ; Bercot, 2006 ; Bonneville et Grosjean, 2009 ; Cott, 1997 ; Ellingson, 2002 ; Grenier, 2007 ; Henry et Grosjean, 2004). Selon Glouberman et Mintzberg (2001, 2002), les organisations de santé sont marquées par les clivages entre quatre "mondes " : le monde médical, le monde de la gestion, le monde institutionnel et le monde non institutionnel. Si les organisations comme les hôpitaux disposent plus de mécanismes pour gérer ces contradictions (par exemple, la structure hiérarchique), l'importance de la négociation est accrue au sein des réseaux de santé, en l'absence d'autorité sur les acteurs-partenaires indépendants (Grosjean, 2004, p. 77). La négociation devient ainsi le « mode de vie » de réseaux de santé (Henry et Grosjean, 2004, p. 236) : s'organisant autour de systèmes de transmission et de partage des informations, ils se maintiennent grâce aux processus de négociation du sens de ces informations (Bercot, 2006, p. 35 ; Bonneville et Grosjean, 2009, p. 94). Au sein des équipes de travail, les acteurs aux pouvoirs inégaux sont impliqués dans la négociation. En étudiant la structure sociale des équipes multidisciplinaires de santé de longue durée, Cott (1997) a remarqué que la prise de décision au sein de ces équipes est réservée aux professionnels occupant les positions hiérarchiques les plus hautes (voir aussi Barley, 1986). Dans cette optique, Bouillon et Maas (2009) ont remis en question le sens même de la notion de collaboration, en soulignant que la conception des acteurs des organisations en tant que collaborateurs " s'apparente à un recadrage de l'organisation, délibérément présentée comme une structure unitaire et cohérente »(Ibid., p. 57) : le " collaborateur " est " une production symbolique managériale à vocation intégratrice » qui « tend à dissimuler la diversité de l'organisation ainsi que les tensions et les contradictions qui la traversent» (Ibid., p. 66).

\section{Axe 2 : Changement dans/des organisations de santé}

La question du changement organisationnel est une thématique clé au moment d'aborder la communication organisationnelle et santé. Deux types de changements font souvent l'objet d'analyse : les changements des modes de gestion et les changements structurels.

1. Changement des modes de gestion. Plusieurs auteurs soulignent que les hôpitaux sont souvent gérés comme des entreprises (Bonneville, 2002 ; Feroni, 2006 ; Gheorghiu et al., 2008). Cette gestion se traduit, entre autres, par « la formalisation des procédures d'activité et la mise en œuvre d'objectifs de qualité ", "l'hyperspécialisation de l'activité médicale ", "la redéfinition de la place et du rôle de différentes catégories professionnelles au sein des institutions » (Feroni, 2006, p. 132), une standardisation accrue (Anderson et al., 2007, p. 293) et la conception du patient en tant que « client » (Mossé et Paradeise, 2003).

Les démarches de qualité transposées du secteur de l'industrie au secteur des soins sont un exemple de ce type de changement (Jolivet, 2011 ; Ogien, 
2009). La mise en œuvre des démarches qualité suppose «l'emprise de la quantification sur l'univers des pratiques hospitalières »(Ogien, 2009, p. 49) et transforme l'organisation et l'évaluation de soins selon une perspective d'efficacité qui repose sur l'élaboration de nouvelles méthodes et techniques normatives visant à encadrer les comportements professionnels (Setbon, 2000, p. 51). Malgré leur déploiement, ces stratégies ne sont pas toujours bien adaptées au contexte hospitalier : elles laissent ressortir les tensions au niveau des valeurs organisationnelles, médicales et gestionnaires, et constituent ainsi une source de contradictions permanentes dans les activités quotidiennes des acteurs (Bercot, 2009 ; Contandriopoulos, 2002 ; Feroni, 2006). L'hôpital se trouve ainsi tiraillé entre deux logiques: celle du "care ", centrée sur les impératifs de la santé et de soins, et celle de l'hôpital-entreprise, guidée par les impératifs de rationalisation, d'encadrement et de contrôle (Arndt et Bigelow, 2000, 2000a ; Gentil, 2013).

2. Changements structurels et nouvelles formes d'organisation. Les changements structurels (par exemple, les fusions, la création de réseaux de santé, les modifications de la structure interne) ont pour but de créer de nouvelles formes organisationnelles afin d'assurer une meilleure coordination, améliorer la qualité des services et en diminuer les coûts. Le leadership collectif et des stratégies décentralisées et participatives ont été prouvées efficaces pour mener à terme les changements au sein des organisations de santé (Denis, 2002 ; Touati et al., 2005 ; Minvielle et Contandriopoulos, 2004).

Les effets contradictoires des changements structurels peuvent être démontrés à l'aide de l'exemple de fusions hospitalières. La hiérarchisation et la standardisation inhérentes aux fusions peuvent être bénéfiques pour les organisations en favorisant la continuité des services et la rationalisation des fonctions administratives, mais peuvent aussi être néfastes pour leur fonctionnement interne, surtout lors de fusions imposées impliquant des « établissements à vocations et cultures différentes " (Richard et al., 2007, p. 159). Suivant un argument similaire, Denis et Langley (2002, p. 151) soulignent que « tandis que le changement structurel fournit le cadre formel pour de nouveaux modes de fonctionnement, les interactions interpersonnelles formelles et informelles au quotidien sont nécessaires pour que les personnes œuvrant dans ces structures puissent inventer ces organisations et apprendre à les faire mieux fonctionner (ou, dit différemment, pour que la culture puisse suivre la structure) ». Or, selon Contandriopoulos (2002, p. 147), « on observe que les pratiques des professionnels ont évolué, que les hôpitaux ont changé, mais le système de soins n'est pas transformé ». L'apport d'une perspective communicationnelle serait ainsi de trouver des réponses organisationnelles à la transformation du travail hospitalier (Raveyre et Ughetto, 2003 ; Mossé et Paradeise, 2003). 


\section{Axe 3 : Gouvernance, régulation et modes de gestion des organisations de santé}

Le concept de gouvernance s'est répandu dans les années 1990, en tant qu'expression de la volonté de transformer la régulation des organisations de santé dans le contexte de «l'augmentation de l'efficacité de la médecine avec le développement de la technologie " et de " la transformation des besoins de santé avec le vieillissement de la population " (Contandriopoulos, 2008, p. 192 sq.). La gouvernance s'exerce à trois niveaux : au niveau macroscopique des gouvernements, au niveau mésoscopique des organisations de santé, et au niveau microscopique des relations entre les acteurs individuels (Id.). «Au niveau de l'organisation de l'offre de soins, ce sont surtout les logiques marchande et technocratique qui sont à l'œuvre " (Contandriopoulos, 2002, p. 145), le but de la gouvernance étant d'améliorer la performance des organisations. Dans une optique communicationnelle, la gouvernance est un objet d'étude intéressant en tant que forme d'action collective qui nécessite une négociation pour que "les décisions prises [...] par un des acteurs du système soient comprises et perçues comme légitimes par tous les autres » (Ibid., p. 146). Barbour et Lammers (2007, p. 201) ont proposé d'analyser la gouvernance et la régulation à quatre niveaux : institutionnel, environnemental, organisationnel et individuel, et de mettre ces niveaux en relation à travers la communication. Dans une perspective interprétative et processuelle, la gouvernance et la régulation ont été conceptualisées comme les processus de « travail de sens » (Jolivet, 2008), qui doivent être compris à travers les pratiques de travail et les narrations des acteurs (Barbour, 2010 ; Harter et al., 2010).

Une des thématiques prédominantes retrouvées dans les textes recensés concerne l'étude des logiques de rationalisation et leur mise en pratique dans les organisations. La manière dont ces logiques participent à la construction du sens de soins basé sur la rationalisation est au cœur du questionnement (Bouillon, 2003, 2005 ; Mayère, 2004, 2004a, 2004b, 2006). La rationalisation est conceptualisée par les auteurs comme triple processus d'optimisation, de codification et de justification des activités. La rationalisation en tant que démarche d'optimisation réfère à une méthode rationnelle mise en œuvre pour assurer la meilleure adéquation possible entre les ressources matérielles, techniques et humaines (Weber, 1995) (par exemple, la standardisation et la normalisation des procédures, la division et la spécialisation du travail, la production d'indicateurs d'évaluation des résultats et de mesure des écarts par rapport aux objectifs). Parallèlement et de manière plus fondamentale, la rationalisation repose sur une démarche de codification, c'est-à-dire d'objectivation de l'organisation par l'usage de l'écriture : "rationaliser » signifie écrire et décrire l'organisation et le social en les mettant en règles, en processus et en procédures formalisées. Enfin, la rationalisation implique un processus de justification, dont l'objectif est d'expliciter et surtout 


\section{$\mathrm{C} \& O \mathrm{n}^{\circ} 45$}

d'argumenter le caractère rationnel des choix réalisés (Boltanski et Thévenot, 1991). Les auteurs se rejoignent pour dire qu'il n'y a pas de rationalisation sans une «méta-rationalisation » (Mayère, 2006), une construction collective du sens qui tend à la naturaliser et à la présenter comme inéluctable (Jolivet, 2010).

\section{Axe 4 : Acteurs et identités professionnelles au sein des organisations de santé}

Dans les travaux recensés, une grande place est accordée aux acteurs clé des organisations de santé comme les médecins, les infirmières, les gestionnaires et les patients. Les deux problématiques suivantes semblent caractériser l'étude des acteurs à partir d'une perspective communicationnelle :

1. Le rôle du patient et les transformations de la relation patient-médecin. La place du patient au sein des organisations de santé évolue avec le dépassement du modèle de paternalisme médical. En France, la notion de démocratie sanitaire suppose une participation active du patient au système de santé et met l'accent sur le respect de libertés fondamentales du patient et son droit à l'information (Rousset, 2009), ainsi que le renforcement de son rôle et sa responsabilisation (Dupagne, 2011). Au Québec, la notion du patientpartenaire s'intègre aux formations de futurs professionnels de la santé, aux nouvelles structures organisationnelles des hôpitaux et au discours entourant le suivi de certaines maladies, particulièrement les maladies chroniques (Dumez et Karazivan, 2011). Dans le contexte de la complexification du système de santé et du développement des réseaux (Grenier, 2007), le patient n'est plus un simple bénéficiaire du système de soins, mais peut y participer en agençant les rapports entre les acteurs (Contandriopolous et al., 2001, p. 42). Ainsi, " la relation de soin est de plus en plus vue comme une coproduction entre les professionnels et les malades " (Bercot et Mayère, 2009, p. 10).

Lintégration des TIC dans le processus de diagnostic et de prestation de soins altère considérablement la relation patient-médecin basée sur l'autorité du médecin sur le plan du savoir et sur le plan social (Béraud, 2002 ; Bonneville, 2002 ; Richard et al., 2007 ; Safran et al., 1998 ; Vitacca et al., 2009). La transformation du rapport patient-médecin rend compte de l'évolution de la place du patient dans l'institution (Gheorghiu et al., 2008), de la redéfinition de la figure du patient qui passe d' "usager " à " client " ou à " citoyen ", selon les cas et les contextes (Mossé et Paradeise, 2003 ; Jolivet et Vasquez, 2011). Cependant, comme le souligne Rousset (2009), la relation classique patient-médecin est toujours très présente au sein des organisations de santé. Bien que le droit à l'information, les TIC et le travail en réseau modifient considérablement les relations entre soignant et soigné, elles « souffrent par nature d'un déséquilibre profond ( Rousset, 2009, p. 38). Nous pouvons toutefois remarquer que ce déséquilibre est compromis par le Web 2.0, « le lieu d'une nouvelle alchimie de la connaissance et des pouvoirs » 
(Dupagne, 2011 : 57). L'e-santé et l'expertise collective qui se construisent au sein de réseaux de patients en ligne ont des impacts non seulement sur la relation entre les patients et les médecins, mais aussi sur la structuration des organisations de santé, «à travers notamment la décentralisation et le passage à une structure horizontale et à une communication aux sens multiples » (Dupagne, 2011 : 59).

2. Tensions dans les pratiques et transformations des identités professionnelles. Les reconfigurations du patient au sein des organisations de soins s'accompagnent d'une redéfinition de différentes catégories professionnelles (Feroni, 2006) et, plus précisément, de la remise en question du sens du travail du médecin : l'identité du médecin basée sur l'expertise est remise en cause dans le contexte du travail en réseau, où les identités sont fondées sur la prestation de services au patient, le travail collectif et le partage de l'information (Grenier, 2007, p. 156 ; Bercot, 2009, p. 54). Dans le contexte de l'émergence d'une double hiérarchie gestionnaire et médicale au sein des organisations de santé (Bercot, 2009), plusieurs chercheurs ont étudié la façon dont les pratiques professionnelles sont négociées et réinventées face aux impératifs de la gestion et aux logiques de rationalisation (Béraud, 2002 ; Groleau et Mayère, 2009; Sainsaulieu, 2006).

L'intégration des TIC au sein des organisations de santé représente un autre facteur susceptible d'altérer la distribution des rôles, transformer les identités professionnelles et questionner la nature et le sens du travail des acteurs (Alasad, 2002). La question centrale est celle de la distribution du travail entre les acteurs humains et les acteurs technologiques, et de la place de ces derniers au sein des équipes de travail (Pirnejad et al., 2008; Safran et al., 1998). L'augmentation de compétences individuelles de certains acteurs grâce à l'utilisation des TIC peut conduire, d'un côté, " à la suppression de certaines tâches, puis de certains métiers » au sein des organisations (Benghozi, 2001, p. 8) et d'un autre côté, à l'émergence de nouvelles tâches et de nouvelles responsabilités (Vitacca et al., 2009, p. 96). Par exemple, nous pouvons mentionner les " coordonnateurs" (infirmières et gestionnaires) qui doivent s'occuper du travail invisible et oublié de coordination et de communication - ce que Strauss (1993) appelle « le travail d'articulation » au sein des organisations de santé qui deviennent de plus en plus complexes et fragmentées (Henry et Grosjean, 2004 ; Lacoste, 2001 ; Raveyre et Ughetto, 2003 ; Schweyer et al., 2004). C'est d'ailleurs sur les nouveaux métiers de coordination et les métiers centrés sur l'information (tels que les webmestres et les managers de l'information) que repose le fonctionnement de réseaux de santé, et un des défis des organisations de santé est de savoir articuler ces métiers aux logiques très différentes (Bourret, 2004).

Dans cette optique, les TIC ont été étudiées en tant qu'acteurs des organisations de santé : à la conception des TIC comme outils s'oppose la conception de la technologie comme acteur prenant une part active au sein 


\section{$\mathrm{C} \& O \mathrm{n}^{\circ} 45$}

des organisations (Benghozi, 2001 ; Berg, 1999 ; Berg et Toussaint, 2003 ; Bonneville, 2002 ; Bruni, 2005 ; Grosjean et Bonneville, 2007). Or, certains chercheurs ont reconnu les outils technologiques en tant que constitutifs du champ de soins et de systèmes de santé (Berg, 1997, 1999 ; Ellingsen et Monteiro, 2003, 2003a, 2003b) et ont fait valoir l'indissociabilité de la technologie et des organisations de santé, qui sont co-construites (Berg et Toussaint, 2003).

\section{Contributions d'une approche de la communication organisationnelle pour l'étude des organisations de santé}

Une première lecture des travaux recensés nous amène à identifier comme caractère distinctif, outre certaines références théoriques et méthodologiques communes mobilisées par les chercheurs, leur portée pratique. Ces travaux s'intéressent en grande majorité à comprendre les enjeux auxquels font quotidiennement face les organisations de santé et leurs acteurs. À partir de cette portée commune, nous distinguons les recherches qui visent des solutions concrètes pour adresser ces enjeux de celles qui s'orientent plutôt dans une démarche heuristique, ou encore celles dont le but est de dénoncer les logiques de pouvoir participant aux phénomènes organisationnels étudiés.

Comme nous l'avons vu dans les pages qui précèdent, l'approche fonctionnaliste (Grosjean \& Bonneville, 2010) est dominante dans les domaines de la communication organisationnelle et de la health communication. Conséquemment, la recherche s'oriente souvent vers une augmentation de la performance de processus organisationnels : améliorer l'interopérabilité des réseaux de coordination inter-organisationnelle; rendre plus performants les systèmes de coopération interne (comme les équipes interdisciplinaires); planifier, mettre en œuvre et légitimer les changements organisationnels; créer des modes de gouvernance permettant de normaliser et de contrôler les pratiques et activités; bien définir les rôles et les relations entre les acteurs, etc. Or, nous croyons que cette approche, de par sa vision instrumentale de la communication (vue en grande partie comme un outil de gestion pour transmettre l'information), réduit la contribution qu'une perspective communicationnelle peut amener à la réflexion sur les organisations de santé. C'est en ce sens que notre recension des écrits a délibérément visé à introduire les approches interprétatives, critiques et constructivistes se basant sur une méthodologie qualitative. Il s'agit ici certes d'un positionnement particulier, le but étant de questionner la nature et le rôle de la communication au-delà des fonctions informatives que nous pouvons lui attribuer.

Dès lors, la communication apparaît sous diverses facettes. Nous la retrouvons sous forme de réseaux d'information entre plusieurs organisations, jouant non seulement un rôle de diffusion, mais aussi de connexion et de mise en commun. La communication devient dans ce sens clé pour « dépasser les logiques individuelles ou d'institutions » et " passer [...] à des pratiques 
coopératives construites autour de chaque patient » (Bourret, 2010, p. 79). La communication apparaît aussi sous la forme de relation entre professionnels de la santé, entre le patient et le médecin, entre les employés et l'organisation. De manière plus précise, la communication est abordée en tant qu'interaction ou conversation entre ces acteurs. La communication en tant que langage (souvent sous sa forme orale) participe ainsi, comme le démontrent bien les travaux de Grosjean et Lacoste (1999), du travail quotidien des acteurs organisationnels, de la manière dont ils " font ensemble ", s'orientent, se comprennent, bref, s'organisent.

Dans la continuité de cette notion d'interaction, nous retrouvons la communication en tant que négociation. Cette idée rejoint les travaux développés par Anselm Strauss (Strauss et al., 1963; Strauss, 1978, 1993) qui rendent compte du travail constant de négociation entre les acteurs d'un hôpital dans le but d'assurer la trajectoire du patient. Strauss soutient que tout ordre social est négocié et que les négociations caractérisent les interactions au sein d'un hôpital. Si l'organisation de santé est un ordre social constamment négocié, c'est que cette négociation s'incarne dans la communication. Il s'agit donc, selon cette perspective, d'une négociation du sens, comprise ici à la fois comme direction et comme signification. Cette dimension symbolique, voire significative, est d'une certaine manière transversale aux problématiques communicationnelles que nous avons soulevées. La question du sens apparaît à la fois dans la mise en cohérence et dans la mise en conflit des différents aspects liés aux organisations de santé. Ainsi, des notions telles que leadership participatif, apprentissage coopératif, changement de valeurs et de cultures, participent du « travail du sens » (Jolivet, 2011) des organisations de santé.

Finalement, le discours apparaît comme dernière forme ou figure de communication évoquée dans les travaux recensés. Plus particulièrement, cette notion est reprise en termes des logiques de la rationalisation : les discours à travers lesquels les organisations de santé sont rationalisées et normalisées. D'une certaine manière, cette notion de discours peut s'assimiler à celle de texte organisationnel (Taylor et Van Every, 2000, 2011) qui s'inscrit dans les normes, les techniques, les procédures, les pratiques organisationnelles et qui est porteur d'une vision rationnelle du travail de soin, et, de manière plus large, de l'organisation de santé en tant qu'entité socialement responsable du dit soin.

Face à cette communication multiforme, nous pouvons définir les organisations de santé comme plurivocales : "il y a une pluralité de voix (celles des politiques, des gestionnaires, des médecins, des aides-soignants, des patients, etc.) qui tendent à se faire entendre " (Grosjean, 2009, p. 193). Dans ce sens, nous trouvons tout à fait pertinente la proposition de Zoller (2010) d'ouvrir la notion d'organisations de santé au-delà des organisations de soins (l'objet d'étude le plus populaire dans les travaux que nous avons recensés), pour y inclure les organisations de promotion et de prévention de 


\section{$\mathrm{C} \& O \mathrm{n}^{\circ} 45$}

la santé, ainsi que toute autre organisation sociale ou relation entre plusieurs organisations qui, d'une manière ou d'une autre, participent de la construction de la santé.

Dans le cadre d'une réflexion communicationnelle sur les organisations de santé, Zoller (2010) consacre, à l'instar de Weick (1995), l'expression " health organizing ", permettant ainsi de mettre la communication au cœur de la constitution même de ce que nous pourrions nommer, en français, le " devenir organisant » de la santé. Ce « devenir organisant » est affecté par de nombreuses transformations et peut s'appréhender à plusieurs niveaux d'analyse, tels les réseaux, les logiques, les interactions et les textes. Dans un même ordre d'idée Real (2010) met en valeur l'apport de la communication organisationnelle à l'étude des organisations de santé et, de manière plus générale, des phénomènes organisationnels liés à la santé. Selon cet auteur, la recherche à la croisée de la health communication et de la communication organisationnelle (ce qu'il propose de nommer health-based organizing), pourrait non seulement contribuer en termes de développement théorique, mais aussi de manière plus pragmatique, permettrait d'améliorer la santé et le bien-être des employés, patients et professionnels de la santé. Aux dires de cet auteur, une perspective de communication organisationnelle et santé serait en mesure d'explorer des solutions aux problèmes et enjeux des pratiques quotidiennes de ceux et celles qui travaillent, visitent, participent à construire les organisations de santé. C'est cette portée pratique et située, basée sur les conversations, les interactions, les récits et autres modalités de la communication qui fait la force de cette approche et qui, nous postulons, gagnerait à être développée davantage. Comme le souligne Mayère (2013, p. 108), « [1]a question d'une approche communicationnelle des organisations de santé s'étend et se recompose ainsi de proche en proche, dessinant un vaste domaine de recherches déjà développées mais aussi encore en bonne part à construire ».

\section{BIBLIOGRAPHIE}

ALASAD J. «Managing technology in the intensive care unit: the nurses' experience », International Journal of Nursing Studies, 39, 2002, p. 407-413.

ANDERSON J. M., TANG S., BLUE C., " Health care reform and the paradox of efficiency: "Writing in" culture ", International Journal of Health Services, 37(2), 2007, p. 291-320.

ARBER A., "Team Meetings in Specialist Palliative Care: Asking Questions as a Strategy Within Interprofessional Interaction ", Qualitative Health Research, 18(10), 2008, p. 1323-1335.

ARNDT M., BIGELOW B., " Commentary: The Potential of Chaos Theory and Complexity Theory for Health Services Management ", Health Care Management Review, 25(1), 2000, p. 35-38. 
ARNDT M., BIGELOW B., "Presenting structural innovation in an institutional environment: hospital's use of impression management », Administrative Science Quarterly, 45(3), 2000a, p. 494-522.

ASH J. S., BERG M., COIERA E., « Some unintended consequences of information technology in health care: the nature of patient care information system-related errors », Journal of the American Medical Informatics Association, 11(2), 2004, p. 104-112.

BARLEY S. R., "Technology as an occasion for structuring: observations on CT scanners and the social order of radiology departments ", Administrative Science Quarterly, 31, 1986, p. 78-108.

BARBOUR J. B., «On the institutional moorings of talk in health care organizations », Management Communication Quarterly, 24(3), 2010, p. 449-456.

BARBOUR J. B., LAMMERS J. C., "Health care institutions, communication, and physicians' experience of managed care : a multilevel analysis ", Management Communication Quarterly, 21(2), 2007, p. 201-231.

BEGUN J. W. et al., "Health care organizations as complex adaptive systems ", in S. M. MICK S. M., WYTTENBACH M, Advances in health care organization theory. San Francisco, Jossey-Bass, 2003, p. 253-288.

BENGHOZI P.-J., «Technologies de l'information et organisation : de la tentation de la flexibilité à la centralisation ", Revue Gestion 2000 (2), 2001, p. 61-80.

BENGHOZI P.-J. et al., Le travail en réseau, au-delà de l'organisation hiérarchique et des technologies de l'information. Paris, L'Harmattan, 2002.

BÉRAUD C., "Les transformations du système de soins au cours des vingt dernières années : point de vue d'un acteur ", Sciences sociales et santé, 20(4), 2002, p. 37-74.

BERCOT R., « La coopération au sein d'un réseau de santé. Négociations, territoires et dynamiques professionnelles ", Négociations, 5(1), 2006, p. 35-49.

BERCOT R., MAYÈRE A., "Organisation et communication au sein des systèmes de santé ». In 77e congrès international ACFAS. Ottawa, Université d'Ottawa, 2009, p. 5-20.

BERCOT R., "Rationaliser la gestion des blocs opératoires : la négociation des territoires et des légitimités professionnelles », Sciences de la société (76), 2009, p. 52-65.

BERG M., "Of forms, containers, and the electronic medical record: some tools for a sociology of the formal ", Science, Technology, and Human Values, 22(4), 1997, p. 403-433.

BERG M., «Accumulating and co-ordinating: occasions for information technologies in medical work », Computer Supported Co-operative Work, 8(4), 1999, p. 373-401.

BERG M., TOUSSAINT P., «The mantra of modelling and the forgotten powers of paper: a sociotechnical view on the development of process-oriented ICT in healthcare ", International Journal of Medical Informatics, 69(2-3), 2003, p. 223-234.

BOLTANSKI L., THÉVENOT L., De la justification. Les économies de la grandeur. Paris, Gallimard, 1991.

BONNEVILLE L., " La réorganisation du système sociosanitaire autour des autoroutes de l'information: Le problème de la productivité », in 2001 Bogues. Globalisme et Pluralisme. Montréal, GRICIS, 2002. 


\section{$\mathrm{C} \& O \mathrm{n}^{\circ} 45$}

BONNEVILLE L., GROSJEAN S., « Les défis que soulève l'informatisation de la pratique médicale sur le plan de l'innovation technologique ", Canadian Journal of Communication (32), 2007, p. 435-456.

BONNEVILLE L., GROSJEAN S., « Mise en place d'un réseau de soins québécois: entre rationalité technique et besoins de communiquer », Sciences de la société (76), 2009, p. 84-97.

BOUILLON J.-L., " Pour une approche communicationnelle des processus de rationalisation cognitive des organisations : contours, enjeux et perspectives ", in $X e$ Colloque bilatéral franco-roumain. Université de Bucarest, 2003. En ligne: archivesic.ccsd. cnrs.fr/docs/00/06/23/71/PDF/sic_00000949.pdf. Consulté le 26 mars 2012.

BOUILLON J.-L., "Autonomie professionnelle et rationalisations cognitives : les paradoxes dissimulés des organisations post-disciplinaires ", Études de communication (28), 2005, p. 91-105.

BOUILLON J.-L., MAAS E., « Figures de l'individu au travail, figures $\mathrm{du}$ "collaborateur" : stratégies face aux rationalisations organisationnelles et communicationnelles ", Communication E̋ Organisation (36), 2009, p. 56-68.

BOURRET C., "Réseaux de santé et nouveaux métiers de l'information ", Documentaliste - Sciences de l'information, 41(3), 2004, p. 174-181.

BOURRET C., "Les réseaux de santé ou la rencontre de la santé et des TIC pour décloisonner le système de santé français », TIC É Société, 1(2), 2008, p. 86-107.

BOURRET C., "Évaluation et communication des nouvelles organisations d'interface : le cas des réseaux de santé ", Communication E Organisation (38), 2010, p. $77-87$.

BOUZON A., "Communication organisationnelle et TIC dans une relation de service en profonde mutation ». In TIC et relations de services dans une économie globale. Castres, XIVe Conférence RESER, 23-24 septembre 2004. En ligne : www.reser.net/ file/4283/. Consulté le 11 octobre 2011.

BRETON M. et al., "L'intégration de la santé publique à la gouverne locale des soins de santé au Québec : enjeux de la rencontre des missions populationnelle et organisationnelle », Pratiques et Organisation des Soins, 39(2), 2008, p. 113-124.

BRUNI A., " Shadowing Software and Clinical Records: On the Ethnography of Non-Humans and Heterogeneous Contexts ». Organization, 12(3), 2005, p. 357-378.

BRUNI A. et al., "Sub-theme 49: Working and Organizing in Technologically Dense Environments ». European Group for Organizational Studies, 2010. En ligne: www.egosnet.org/jart/prj3/egos/main.jart? rel=de\&reserve-mode=active\&contentid=1332406479056\&subtheme_id=1277261035048. Consulté le 2 avril 2012.

CARONNA C. A., "Why Use Qualitative Methods to Study Health Care Organizations? Insights from Multi-Level Case Studies ». In BOURGEAULT I et al. (ed.), The SAGE Handbook of Qualitative Methods in Health Research. London: Sage, 2010, p. 71-87.

CONTANDRIOPOULOS A.-P., " Pourquoi est-il si difficile de faire ce qui est souhaitable? » Gestion, 27(3), 2002, p. 142-150. 
CONTANDRIOPOULOS A.-P., « La gouvernance dans le domaine de la santé : une régulation orientée par la performance », Santé publique, 20(2), 2008, p. 191-199.

COOREN F. et al., "Communication, organizing, and organization: An overview and introduction to the special issue ", Organization Studies, 32(9), 2011, p. 1149-1170.

COTT C., "We decide, you carry it out”: A social network analysis of multidisciplinary long-term care teams ", Social Science Medicine, 45(9), 1997, p. 1411-1421.

DENIS J. L., "Gouvernance and management of change in Canada's health system ", Commission sur l'avenir des soins de santé au Canada, Étude no 36, 2002. En ligne : http:// publications.gc.ca/collections/Collection/CP32-79-36-2002E.pdf. Consulté le 11 octobre 2011.

DENIS J. L. et al., " Peut-on transformer les anarchies organisées? Leadership et changement radical dans un hôpital ", Ruptures, revue transdisciplinaire en santé, 2(2), 1995, p. 165-189.

DENIS J. L. et al., "The dynamic of collective leadership and strategic change in pluralistic organizations ", Academy of Management Journal (44), 2001, p. 809-837.

DENIS J. L. et al., "Strategizing in pluralistic contexts: Rethinking theoretical frames ", Human Relations, 60(1), 2007, p. 179-215.

DENIS J. L., LANGLEY A., " Faut-il changer nos façons de changer? » Gestion, 27(3), 2002, p. 151-152.

DUMEZ V., KARAZIVAN P., «L'usager bras droit du médecin ». Montéal, Bureau de l'expertise patient partenaire de l'Université de Montréal, 2011. En ligne : www.rpcu. qc.ca/pdf/forum2011/cds.pdf, consulté le 10 mars 2012.

DUPAGNE D., « E-santé », Communications, 88(1), 2011, p. 57-65.

ELLINGSEN G., MONTEIRO F., « A patchwork planet. Integration and cooperation in hospitals ", Computer Supported Cooperative Work, 12(1), 2003, p. 71-95.

ELLINGSEN G., MONTEIRO F., « Big is beautiful: electronic patient records in Norway, 1980-2000 ", Methods of Information in Medicine, 42(4), 2003a, p. 366-370.

ELLINGSEN G., MONTEIRO F., « Mechanisms for producing a working knowledge: enacting, orchestrating, and organizing ", Information and Organization, 13(3), 2003b, p. 203-229.

ELLINGSON L. L., "Communication, Collaboration, and Teamwork among Health Care Professionals », Communication Research Trends, 21(3), 2002, p. 3-21.

FERONI I., "Mutations institutionnelles, évolution de l'activité : le contexte des restructurations hospitalières des années quatre-vingt-dix ", Sciences de la société, 24(4), 2006, p. 129-134.

GENTIL S., «Les 'agencements organisationnels' des situations perturbées : la coordination d'un bloc opératoire à la pointe de la rationalisation industrielle ", Revue internationale de la communication sociale et publique, 8, 2013, p. 65-80.

GHEORGHIU M. D. et al., "Les changements organisationnels et leurs outils : entretiens avec les dirigeants d'hôpitaux ", Centre d'études de l'emploi, Document de travail $n^{\circ} 107,2008$. En ligne : http://www.cee-recherche.fr/fr/doctrav/107organisationnel_outils_entretiens_dirigeants_hopitaux.pdf. Consulté le 11 octobre 2011. 


\section{$\mathrm{C} \& O \mathrm{n}^{\circ} 45$}

GLOUBERMAN S., MINTZBERG H., « Gérer les soins de santé et le traitement de la maladie », Gestion, 27(3), 2002, p. 12-22.

GRENIER C., "Le difficile apprentissage de la coordination dans les réseaux de santé », in SAINT LAURENT-KOGAN A.-F., METZGER J.-L. (dir.), Où va le travail à l'ère du numérique? Paris, Presses de l'École de Mines, 2007, p. 147-166

GROLEAU C., MAYĖRE A., « Médecins avec ou sans frontière: contradiction et transformation des pratiques professionnelles ", Sciences de la société (76), 2009, p. 66-83.

GROSJEAN M., « La négociation constitutive et instituante. Les co-configurations du service en réseau de soins ", Négociations, 2(2), 2004, p. 75-90.

GROSJEAN M., LACOSTE M., Communication et intelligence collective : le travail à l’hôpital. Paris, Presses Universitaires de France, 1999.

GROSJEAN S., "Communication dans un centre de répartition des urgences 911 », Canadian Journal of Communication (33), 2008, p. 10-120.

GROSJEAN S., « Saisir la texture des organisations du système de la santé ». Ottawa, Colloque de l'ACFAS « Organisation et communication au sein des systèmes de santé », 11-12 mai 2009.

GROSJEAN S., BONNEVILLE L., " Logiques d'implantation des TIC dans le secteur de la santé ", Revue française de gestion, 172(3), 2007, p. 145-157.

HARTER L. M. et al., " Narrating "New Normals" in Health Care Contsexts ». Management Communication Quarterly, 24(3), 2010, p. 465-473.

HENRY J., GROSJEAN M., « La négociation au coeur du travail : l'exemple du travail en réseau », in GROSJEAN M., MONDADA L. (dir.), La négociation au travail. Presses Universitaires de Lyon, 2004, p. 219-237.

JOLIVET A., «Voir les normes comme un travail de sens : le texte comme agencement de narrations ", Études de communication (34), 2010, p. 75-92.

JOLIVET A., La certification à l'épreuve de la communication : figures de la qualité et travail du sens. Le cas d'un établissement de santé. Thèse de doctorat en Sciences de l'Information et de la Communication, Université Toulouse, 2011.

JOLIVET A., VÁSQUEZ C., "Reconfiguration de l'organisation : suivre à la trace les figures textualisées - le cas de la figure du patient ", Études de communication (36), 2011, p. 129-146.

LACOSTE M., «Quand communiquer c'est coordonner. Communication à l'hôpital et coordination des équipes ", in BORZIEUX A., FRAENKEL B. (dir.), Langage et travail : communication, cognition, action. Paris, CNRS Éditions, 2001, p. 323-342.

MAYÈRE A., «Rationalisation de la communication et organisations contemporaines: le cas de projets d'implantation de PGI/ERP ", Communication E Organisation, 24(1), 2004, p. 363-379.

MAYÈRE A., «Formalisation des savoirs : tensions et enjeux dans les organisations contemporaines ». In METZGER J.-P. (dir.), Le partage des savoirs. Logiques, contraintes et crises. Paris, l'Harmattan, 2004a, p. 83-100.

MAYÈRE A., "Les ERP comme projet de rationalisation des productions d'information et des communications à l'échelle de groupe mondialisés. Question de sens, 
de liens, de pouvoir ». In Questionner l'internationalisation : cultures, acteurs, organisations, machines. Béziers: XIV congrès national des SFSIC, 2-4 juin 2004b, p. 635-641.

MAYÈRE A., «Repenser les TIC: Pour un cadre d'analyse des évolutions des dispositifs d'information - communication et des pratiques professionnelles ». Communication au colloque international « Pratiques et usages organisationnels des sciences et technologies de l'information et de la communication », Rennes, 2006.

MAYÈRE A., "Communication organisationnelle et organisations de santé : enjeux et perspectives ", Revue internationale de la communication sociale et publique, 8, 2013, p. 99-111.

MINVIELLE É, CONTANDRIOPOULOS A. P., « La conduite du changement : Quelles leçons tirer de la restructuration hospitalière? " Revue française de gestion, 150, 2004, p. 29-53.

MINTZBERG H., GLOUBERMAN S., " Managing the Care of Health and the Cure of Disease-Part II: Integration », Health Care Management Review, winter 2001, p. 72-86.

MOSSÉ P., PARADEISE C., "Restructurations de l'hôpital ; recompositions des hôpitaux. Réflexions sur un programme », Revue Française des Affaires sociales, 3(3), 2003, p. 143-155.

OGIEN A., "L'hôpital saisi par la qualification. Une analyse de l'usage gestionnaire de la notion de qualité », Sciences de la société (76), 2009, p. 30-51.

PIRNEJAD H. et al., "Intra-Organizational Communication in Healthcare ", Methods of Information in Medicine, 4(47), 2008, p. 336-345.

RAVEYRE M., UGHETTO P., "Le travail, part oubliée des restructurations hospitalières », Revue Française des Affaires sociales (3), 2003, p. 95-119.

REAL K., "Health-related organizational communication : a general platform for interdisciplinary research ", Management Communication Quarterly, 24(3), 2010, p. 457464.

RICHARD M.-C. et al., "La fusion des établissements de santé et de services sociaux : recension des écrits sur ses enjeux et ses impacts ", Nouvelles pratiques sociales, 20(1), 2007, p. 151-164.

ROBEY D., BOURDEAU M.-C., « Accounting for the contradictory organizational consequences of information technology: theoretical directions and methodological implications ", Information Systems Research, 10(2), 1999, p. 167-185.

ROUSSET G., "Quelle place pour l'usager dans le système de santé français? ", in 77e congrès international ACFAS, Organisation et communication au sein des systèmes de santé. Ottawa, Université d'Ottawa, 2009, p. 34-46.

SAFRAN C. et al., "Electronic communication and collaboration in a health care practice ", Artificial Intelligence in Medicine (12), 1998, p. 137-151.

SAINSAULIEU I., La communauté de soins en question. Le travail hospitalier face aux enjeux de la société. Rueil-Malmaison, Éditions Lamarre, 2006.

SCHWEYER F.-X. et al., Créer et piloter un réseau de santé. Un outil de travail pour les équipes. Paris, Éditions de l'École nationale de la santé publique, 2004. 


\section{$C \& O n^{\circ} 45$}

SETBON M., "La qualité des soins, nouveau paradigme de l'action collective ? ", Sociologie du travail (42), 2000, p. 51-68.

SHEAFF R. et al., "A subtle gouvernance: "soft" medical leadership in English primary care ", Sociology of Health E' Illness, 25(5), 2003, p. 408-428.

STRAUSS A., Negotiations: Varieties, Contexts, Processes, and Social Order. San Francisco, Jossey-Bass, 1978.

STRAUSS A., Continual Permutation of action. New York, Aldine de Gruyter, 1993.

STRAUSS A. et al., "The hospital and its negotiated order ", in FRIEDSON E. (dir.), The Hospital in Modern Society. New York, Free Press, 1963, p. 147-169.

TAYLOR J. R., VAN EVERY E. J., The emergent organization. Communication as its site and surface. London, Lawrence Erlbaum, 2000.

TAYLOR J. R., VAN EVERY E. J., The situated organization: Case studies in the pragmatics of communication research. New York, Routledge, 2011.

TOUATI N. et al., "Introduire le changement dans les systèmes de soins au Québec : comment tirer profit de l'expérimentation sociale? ", Sciences sociales et santé, 23(2), 2005, p. $75-102$

VÁSQUEZ C., JOLIVET A., « La santé reconfigurée et reconfigurante : de la valeur à la norme ", Revue internationale de la communication sociale et publique, 8, 2013, p. I-XIII.

VITACCA M. et al., "Socio-technical and organizational challenges to wider e-Health implementation ", Chronic Respiratory Disease (6), 2009, p. 91-97.

WEBER M., Économie et société. Paris, Éditions Plon Agora, 1995.

WEICK K. E., "South Canyon Revisited : Lessons from High Reliability Organizations», Wildfire (4), 1995, p. 54-68.

WEICK K. E., ROBERTS K. H., « Collective Mind in organizations : Heedful interrelating on flight decks », Administrative Science Quarterly (38), 1993, p. 357-381.

WEICK K. E., SUTCLIFFE K. M., Managing the unexpected : Assuring High performance in an age of complexity. San Francisco, Jossey-Bass, 2001.

ZOLLER H. M., "What are health organizations? ", Management Communication Quarterly, 24(3), 2010, p. 482-490.

Résumé : Dans le cadre d'une réflexion sur les organisations de santé, nous présentons les résultats d'une revue de littérature portant sur les dynamiques organisationnelles et communicationnelles qui caractérisent ces organisations. Cette thématique de recherche, qui relève de ce que nous proposons de nommer la « communication organisationnelle et santé ", est définie par des problématiques particulières, ainsi que par sa portée pratique, c'est-à-dire par la volonté de comprendre les enjeux auxquels font quotidiennement face les organisations de santé et proposer des démarches et interprétations pour les adresser. En réfléchissant aux orientations de la recherche dans ce domaine et en nous basant sur une approche constitutive de la communication organisationnelle, nous proposons d'élargir la définition d'organisation de santé aux processus organisants qui en seraient constitutifs, soit le « health organizing ». 
Mots-clés : organisation de santé, communication organisationnelle, approches constitutives de la communication organisationnelle, health organizing.

Abstract : As part of an inquiry into healthcare organizations, we present the results of a literature review on organizational and communicational dynamics that characterize these organizations. This research theme, which we propose to call 'organizational communication and health', is defined by some specific issues, as well by its practical orientation, namely, the willingness to understand the day-to-day problems faced by health organizations and to suggest appropriate approaches and interpretations to address them. Reflecting on these research directions and drawing on a constitutive approach to organizational communication, we suggest to broaden the definition of health organizations to the organizing processes that constitute healthcare systems, that is 'bealth organizing'.

Keywords : health organizations, organizational communication, constitutive approaches to organizational communication, health organizing

\section{Annexe : Synthèse des axes thématiques}

\begin{tabular}{|c|c|c|}
\hline Axe thématique & Problématiques & Quelques auteur-e-s \\
\hline $\begin{array}{l}\text { Coordination et } \\
\text { collaboration aux } \\
\text { niveaux intra- et } \\
\text { inter-organisationnels }\end{array}$ & $\begin{array}{l}\text { - La constitution et } \\
\text { la distribution des } \\
\text { savoirs } \\
\text { - Le réseau comme } \\
\text { mode spécifique de } \\
\text { coordination des } \\
\text { organisations de } \\
\text { santé } \\
\text { - La négociation } \\
\text { et les rapports de } \\
\text { pouvoir }\end{array}$ & $\begin{array}{l}\text { Arber (2008), Ash et coll. (2004), Barley } \\
\text { (1986), Benghozi (2001), Benghozi et coll. } \\
\text { (2002), Bercot (2006), Bercot et Mayère (2009), } \\
\text { Bonneville et Grosjean (2009), Bouillon et } \\
\text { Maas (2009), Bourret (2008, 2010), Bouzon } \\
\text { (2004), Breton et coll. (2008), Cott (1997), } \\
\text { Ellingson (2002), Gheorghui et Moatty } \\
\text { (2006), Glouberman et Mintzberg (2001, } \\
\text { 2002), Grenier (2007), Grosjean (2004, 2008), } \\
\text { Grosjean et Bonneville (2007, 2009), Henry et } \\
\text { Grosjean (2004), Lacoste (2001), Pirnejad et } \\
\text { coll. (2008), Robey et Boudreau (1999), Safran } \\
\text { et coll. (1998), Schweyer et coll. (2004), Sheaff } \\
\text { et coll. (2003), Vitacca et coll. (2009) }\end{array}$ \\
\hline $\begin{array}{l}\text { Changement des/ } \\
\text { dans les organisations } \\
\text { de santé }\end{array}$ & $\begin{array}{l}\text { - Changement des } \\
\text { modes de gestion } \\
\text { - Changements } \\
\text { structurels et } \\
\text { nouvelles formes } \\
\text { organisationnelles }\end{array}$ & $\begin{array}{l}\text { Anderson et coll. (2007), Arndt et Bigelow } \\
\text { (2000a), Béraud (2002), Bercot (2009), } \\
\text { Bonneville (2002), Contandriopoulos (2002), } \\
\text { Denis (2002), Denis et Langley (2002), } \\
\text { Feroni (2006), Gentil (2013), Gheorghui } \\
\text { et coll. (2008), Jolivet (2011), Minvielle et } \\
\text { Contandriopoulos (2004), Mossé et Paradeise } \\
\text { (2003), Ogien (2009), Raveyere et Ughetto } \\
\text { (2003), Richard et coll. (2007), Setbon } \\
\text { (2000), Touati et coll. (2005) }\end{array}$ \\
\hline
\end{tabular}




\begin{tabular}{|c|c|c|}
\hline Axe thématique & Problématiques & Quelques auteur-e-s \\
\hline $\begin{array}{l}\text { Gouvernance, } \\
\text { régulation et modes } \\
\text { de gestion des } \\
\text { organisations de santé }\end{array}$ & $\begin{array}{l}\text { - Les logiques de } \\
\text { rationalisation }\end{array}$ & $\begin{array}{l}\text { Barbour (2010), Barbour \& Lammers (2007), } \\
\text { Boltanski et Thévenot (1991), Bouillon } \\
\text { (2003, 2005), Contandriopoulos (2002, } \\
\text { 2008), Harter (2010), Jolivet (2010), Mayère } \\
\text { (2004, 2004a, 2004b, 2006), Weber (1995) }\end{array}$ \\
\hline $\begin{array}{l}\text { Acteurs et identités } \\
\text { professionnelles au } \\
\text { sein des organisations } \\
\text { de santé }\end{array}$ & $\begin{array}{l}\text { - Le rôle du patient } \\
\text { et la transformation } \\
\text { de la relation } \\
\text { patient-médecin } \\
\text { - Tensions dans } \\
\text { les pratiques et } \\
\text { transformations } \\
\text { des identités } \\
\text { professionnelles }\end{array}$ & $\begin{array}{l}\text { Alasad (2002), Benghozi (2001), Béranger } \\
\text { (2012), Béraud (2002), Bercot (2006, 2009), } \\
\text { Bercot et Mayère (2009), Berg (1997, 1999), } \\
\text { Berg et Toussaint (2003), Bonneville (2002), } \\
\text { Bourret (2004), Bruni (2005), Dumez et } \\
\text { Karazivan (2011), Dupagne (2011), Ellingsen } \\
\text { et Monteiro (2003, 2003a, 2003b), Feroni } \\
\text { (2006), Gheorghiu et coll. (2008), Grenier } \\
\text { (2007), Groleau et Mayère (2009), Grosjean } \\
\text { et Bonneville (2007), Henry et Grosjean } \\
\text { (2004), Jolivet et Vásquez (2011), La Peyre } \\
\text { et Le Fleuve (2005), Lacoste (2001), Mossé } \\
\text { et Paradeise (2003), Pirnejad et coll. (2008), } \\
\text { Raveyre et Ughetto (2003), Richard et coll. } \\
\text { (2007), Rousset (2009), Safran et coll. (1998), } \\
\text { Sainsaulieu (2006), Schweyer et coll. (2004), } \\
\text { Strauss (1978, 1993), Strauss et coll. (1963), } \\
\text { Vitacca et coll. (2009) }\end{array}$ \\
\hline
\end{tabular}

